\title{
Criminologie
}

\section{Repartir à zéro loin de sa terre natale : la réinstallation des Lost Boys and Girls of Sudan au Canada}

\section{Robynn Collins}

Volume 45, numéro 1, printemps 2012

Violence politique et conflits armés

URI : https://id.erudit.org/iderudit/1008376ar

DOI : https://doi.org/10.7202/1008376ar

Aller au sommaire du numéro

Éditeur(s)

Les Presses de l’Université de Montréal

ISSN

0316-0041 (imprimé)

1492-1367 (numérique)

Découvrir la revue

Citer cet article

Collins, R. (2012). Repartir à zéro loin de sa terre natale : la réinstallation des Lost Boys and Girls of Sudan au Canada. Criminologie, 45(1), 51-69.

https://doi.org/10.7202/1008376ar
Résumé de l'article

Les études sur les jeunes survivants de conflits armés et de violence politique viennent approfondir un domaine d'intérêt grandissant en criminologie. À cet égard, cet article met en lumière comment certains facteurs de protection ont pu favoriser la réinstallation au Canada d'une trentaine de «Lost Boys and Girls of Sudan ", des jeunes réfugiés séparés de leurs familles en raison de la guerre au Sud-Soudan. L'analyse préliminaire d'entrevues individuelles et de groupes de discussion menés avec ces jeunes permet d'éclairer le rôle joué par des facteurs de protection qu'ils ont eux-mêmes cernés, notamment le désir de devenir de " bons citoyens ", leur débrouillardise et leurs perspectives d'avenir, la culpabilité du survivant et le sentiment de responsabilité envers le pays d'origine, les familles de substitution et le capital affectif, le capital social et religieux ainsi que l'étiquette même de "Lost Boys and Girls ».
Ce document est protégé par la loi sur le droit d'auteur. L'utilisation des services d’Érudit (y compris la reproduction) est assujettie à sa politique d'utilisation que vous pouvez consulter en ligne.

https://apropos.erudit.org/fr/usagers/politique-dutilisation/ 


\title{
Repartir à zéro loin de sa terre natale: la réinstallation des Lost Boys and Girls of Sudan au Canada
}

\author{
Robynn Collins \\ Doctorante \\ Département de criminologie, Université d'Ottawa \\ robynn.farrah@gmail.com
}

RÉSUMÉ - Les études sur les jeunes survivants de conflits armés et de violence politique viennent approfondir un domaine d'intérêt grandissant en criminologie. À cet égard, cet article met en lumière comment certains facteurs de protection ont pu favoriser la réinstallation au Canada d'une trentaine de "Lost Boys and Girls of Sudan», des jeunes réfugiés séparés de leurs familles en raison de la guerre au Sud-Soudan. L'analyse préliminaire d'entrevues individuelles et de groupes de discussion menés avec ces jeunes permet d'éclairer le rôle joué par des facteurs de protection qu'ils ont eux-mêmes cernés, notamment le désir de devenir de "bons citoyens», leur débrouillardise et leurs perspectives d'avenir, la culpabilité du survivant et le sentiment de responsabilité envers le pays d'origine, les familles de substitution et le capital affectif, le capital social et religieux ainsi que l'étiquette même de "Lost Boys and Girls».

MOTS-CLÉS - Lost Boys and Girls of Sudan, réfugiés séparés/non accompagnés, criminologie, facteur de protection, immigration, Canada.

\section{Introduction}

En 1983, une guerre civile a éclaté lorsque les extrémistes islamiques du nord du Soudan ont envahi et attaqué les villages chrétiens du SudSoudan. Plusieurs hommes et femmes ont été massacrés durant ce profond conflit ethnico-religieux qui a duré plusieurs décennies. Entre 17000 et 25000 mineurs non accompagnés ont fui leur village (Refugees International: 2002). De 1984 à 2001, ces jeunes garçons et ces jeunes filles ont parcouru des milliers de kilomètres à pied, littéralement perdus

Traduit de l'anglais par Élise Chapdelaine et édité par Joanne Desroches. 
dans la brousse, et ont parfois été recrutés en chemin dans les armées. Après plus d'une décennie à se déplacer en pleine guerre et à se débrouiller par eux-mêmes, ceux qui n'ont pas été tués ou mutilés en cours de route par les animaux sauvages se sont retrouvés dans les camps de réfugiés au Kenya et en Éthiopie durant les années 1991 à 2002. Dans les camps de réfugiés, les garçons ont été autorisés à vivre dans leur propre partie du camp - ce qui était même attendu d'eux -, où ils avaient leurs propres habitations, et où chacun avait son rôle bien défini dans le groupe. Les garçons étaient organisés en collectif et du counseling leur était offert par des travailleurs humanitaires. La plupart des filles mineures non accompagnées ont été placées dans des familles « d'accueil» qui s'attendaient à ce qu'elles assument des tâches traditionnelles telles que faire la cuisine, le ménage, ramasser du bois et prendre soin des jeunes enfants (Matheson, 7 juin 2002; Newland, 2004).

Dans les camps de réfugiés, le Haut Commissariat des Nations Unies pour les réfugiés (HCNUR) a lancé un programme d'établissement afin de rediriger les jeunes de ces camps appauvris et surpeuplés vers des pays développés comme les États-Unis, l'Australie et le Canada. Des listes établies par le conseiller de l'organisme ont ensuite été utilisées pour recenser les candidats à la réinstallation (McKelvey, 3 octobre 2003; HCNUR, 2002). Sur environ 3700 mineurs non accompagnés qui se sont réinstallés aux États-Unis, seulement 89 étaient des femmes (Newland, 2004). Au moment où les programmes de réinstallation ont débuté, la plupart des filles avaient pratiquement «disparu» dans des familles d'accueil, étaient enceintes, avaient été vendues comme épouses, ou étaient utilisées comme domestiques et servantes (Newland, 2004: 9). Certaines jeunes filles ont cependant réussi à sortir des camps de réfugiés et se sont réinstallées au Canada. Tous ces jeunes sont légalement connus sous le nom de mineurs «non accompagnés» ou «séparés», et au cours des deux dernières années, nous avons eu l'occasion de nous familiariser avec plus d'une trentaine d'entre eux qui ont été séparés de leurs familles et qui sont venus au Canada en provenance du Soudan ${ }^{1}$.

Puisque de nombreuses études inspirées du concept de désorganisation sociale (voir entre autres Shaw et McKay, 1969; Burisk, 1989; Hagen, 1994; Stowell et Martinez, 2007) établissent un lien entre le

1. Je tiens à remercier mes superviseurs, Dre Sylvie Frigon et Dre Denise Spitzer, ainsi que Dr Victor da Rosa à l'Université d'Ottawa pour leur orientation et des directives en ce qui concerne cette recherche, et Dre Maritza Felices-Luna pour son soutien dans le processus de publication. 
fait d'être un jeune immigrant et le risque de devenir délinquant, notre intérêt s'est porté sur comment ces jeunes considèrent avoir surmonté les failles des systèmes sociaux (comme le système d'éducation) et ainsi échappé aux comportements criminels et à la criminalité.

\section{Études précédentes}

Les mineurs séparés par la guerre courent des risques accrus d'avoir des problèmes de santé fonctionnelle, comportementale et mentale dans leur pays de réinstallation (Beiser et al., 1995; Duncan 2001; Geltman et al., 2005). Une recherche sur la santé mentale de 63 mineurs séparés soudanais réfugiés en Australie et demandeurs d'asile, a révélé seize événements traumatisants qui ont été directement ou indirectement vécus par les participants, tels que la séparation forcée des membres de la famille, l'assassinat d'un parent, le manque de nourriture et d'eau, une situation de combat, être perdus ou enlevés, le viol ou l'abus sexuel (Schweitzer et al., 2006). Tous les Lost Boys and Girls dans cette étude ont vécu certains de ces événements traumatisants, voire tous.

À leur tour, Geltman et al. (2005) ont étudié la santé fonctionnelle et comportementale de 304 mineurs séparés placés en famille d'accueil (un an après leur établissement aux États-Unis) et ont constaté que 20\% d'entre eux ont reçu un diagnostique de syndrome du stress posttraumatique, auquel ont été corrélés l'isolement social et des antécédents de blessures. De même, dans sa recherche sur l'adaptation de 1125 Lost Boys of Sudan réinstallés aux États-Unis, Duncan (2001) a constaté que plusieurs d'entre eux ressentaient des symptômes significatifs de détresse psychologique; presque tous éprouvaient des symptômes modérés ou graves de traumatismes non résolus, plusieurs souffraient d'anxiété troublant leur vie quotidienne, et bien qu'ils aient été rares à souffrir de dépression ou à avoir des sentiments de culpabilité, l'auteur a observé que les filles avaient tendance à être plus déprimées que les garçons (Duncan: 2). Néanmoins, en dépit des nombreux symptômes et problèmes de santé mentale observés dans ce groupe, Duncan n'y a décelé aucun comportement de délinquance, de toxicomanie, de troubles d'apprentissage ou de mémoire, ou de somatisation. Des études récentes ont également déterminé des facteurs de cohésion chez les mineurs séparés, dont la famille, les amis et les relations sociales positives, la collectivité et le soi collectif (Goodman, 2004; Nhial et Mills, 2004). 
Aux lendemains de conflits déclarés et de violence militaire, les jeunes ont tendance à considérer la sécurité et l'éducation comme leurs principales priorités (USAID, 2004: 9). Il existe toutefois des défis d'ordre éducatif propres aux jeunes réfugiés au Canada, qui rendent ces derniers particulièrement vulnérables à certains problèmes (Yau, 1995). Comme l'a révélé l'étude de Yau, les mineurs séparés risquent plus de connaître des problèmes liés à l'éducation en raison de plusieurs facteurs, notamment le peu ou l'absence totale de scolarité officielle antérieure, la tendance à rester loin des figures d'autorité, et le stress post-traumatique. D'autres études ont conclu que les défis éducatifs qu'affrontent les enfants réfugiés les exposent à un risque élevé d'échec à leurs cours, voire de décrochage scolaire complet (Anisef et Kilbride, 2001 ; Anisef et al., 2001).

Certains chercheurs ont cerné des facteurs de protection agissant à titre de forces de cohésion et favorisant la résilience des mineurs séparés par la guerre, dont voici quelques exemples : l'affirmation de leur allégeance et croyances religieuses pour donner sens à leur expérience, l'éducation comme moyen de développer leur estime de soi, ainsi que leur désir de devenir des membres actifs de la société (Duncan 2001: 4).

Les jeunes réfugiés séparés sont le sujet d'encore très peu d'études (Ali et al., 2002). Cet article décrit brièvement comment un groupe d'enfants réfugiés séparés a vécu l'expérience de leur prise en charge depuis leur arrivée au Canada et donne un aperçu des connaissances et des pratiques auxquelles ils ont eu recours pour réussir. Bien que cette contribution fasse partie d'une étude beaucoup plus large sur comment la résilience et le capital social sont construits, contrôlés et entretenus par les jeunes réfugiés séparés pour surmonter les risques auxquels ils doivent faire face, elle se concentre ici sur un ensemble de pratiques qui les ont aidés dans leur lutte acharnée pour maximiser toutes leurs chances lors du processus de rétablissement.

\section{Méthodologie et réflexions éthiques}

Nous avons été invitée par une communauté de mineurs séparés soudanais à nous pencher sur leur histoire après avoir rédigé un billet sur un blogue traitant de la jeunesse et la violence politique. Nous avons reçu quelques réponses des Lost Boys of Sudan qui ont suscité notre intérêt et qui nous ont permis d'entamer un dialogue avec quelques-uns 
d'entre eux. Nous étions tellement intriguée par tous les obstacles qu'ils ont rencontrés et les possibilités qui se sont présentées à eux au cours de leur long voyage vers le Canada que nous avons décidé de changer notre sujet de recherche et de travailler avec cette communauté pour en apprendre davantage sur leur histoire et documenter, grâce à leurs témoignages, comment les jeunes réfugiés gèrent le processus de réinstallation au Canada. Pour y parvenir, une méthode d'échantillonnage en boule de neige a été utilisée. Tous les participants à notre étude sont des adultes âgés de 19 à 33 ans, venus au Canada entre 2001-2006 en provenance du Soudan. Tous ont demandé le statut de réfugié et se sont identifiés en tant que Lost Boys and Girls of Sudan. Quelques-uns ont été formés en tant qu'enfants soldats, certains ont combattu sur le front (d'autres ont vécu les deux), mais tous ont été séparés de leur famille alors qu'ils étaient âgés de moins de 18 ans.

La collecte des données a eu lieu au moyen de groupes de discussion et d'entrevues individuelles. Quatre groupes de discussion se sont tenus à Calgary et à Edmonton en 2007, et chacune des séances a réuni de huit à quinze participants pendant deux à trois heures. Le but de ces groupes était d'explorer quatre grands thèmes - les défis et les possibilités; les attentes; les responsabilités et les pressions; ainsi que la dynamique identitaire - qui ont ensuite été utilisés pour concevoir un questionnaire pour les entretiens individuels. Des entretiens semistructurés et en profondeur ont aussi été réalisés avec 19 participants (8 femmes et 11 hommes) à Calgary et à Edmonton en 2009 (en sessions de $1 \frac{1}{2}$ à $2 \frac{1}{2}$ heures). Nous avons utilisé un guide d'entrevue pour comprendre comment les participants expliquent le succès ou l'échec de leur réinstallation au Canada, comment ils interprètent les risques et la résilience dans leur vie, et comment ils construisent ou maintiennent leur capital social. Chaque participant s'est vu attribuer un pseudonyme dans le but de préserver l'anonymat. Tous les groupes de discussion et les entretiens individuels ont été retranscrits et les participants ont reçu une copie de la transcription de l'entretien pour leur permettre d'apporter des corrections ou des changements, si nécessaire. Une approche inductive a été utilisée pour déceler les tendances et les thèmes qui ont émergé. Le logiciel de traitement des données NVivo8 s'est avéré très utile pour gérer une multitude d'informations, explorer les relations entre les thèmes et établir de nouveaux liens entre les concepts. Nous avons manuellement encodé chaque entrevue et créé des arborescences de nœuds hiérarchisés pour regrouper les thèmes. 
Cette étude comporte certaines limites éthiques et opérationnelles comme celle de n'avoir consulté que des réfugiés séparés soudanais qui parlaient l'anglais. Pour cette raison, les thèmes qui sont ressortis des discussions sur l'expérience de réinstallation (comme le risque, le capital social et la résilience) seraient vraisemblablement très différents si nous avions consulté des réfugiés qui ne parlaient pas l'anglais avant leur migration, qui n'ont pas eu les ressources pour l'apprendre après, ou des réfugiés pour qui l'accès à l'éducation, l'emploi et un réseau social étaient plus difficiles. On pourrait considérer qu'il s'agit d'un échantillon «aberrant», selon le terme employé par Gladwell (2008) pour qualifier les échantillons qui s'écartent extraordinairement de la norme. De plus, on pourrait dire que ces enfants réfugiés sont «la crème de la crème» puisqu'ils se révèlent d'extraordinaires exceptions à la norme en raison de leur expérience de la guerre, et non pas en dépit de celle-ci. Cette étude se limite à la compréhension des connaissances et des pratiques d'un groupe d'enfants séparés soudanais touchés par la guerre et vivant au Canada. En raison de l'objectif exploratoire de cette recherche, les résultats de l'étude ne peuvent être généralisés à tous les enfants réfugiés séparés.

Il est devenu évident que pour travailler avec cette population, un chercheur se devait de suivre certains protocoles implicites. Des nombreux défis inhérents à ce projet, le plus délicat a été celui de développer et de maintenir une bonne relation de travail avec les «gardiens» du groupe. Les gardiens sont des individus qui peuvent maîtriser l'accès aux membres de la communauté officiellement (comme un gardien de prison) ou implicitement (comme un leader autoproclamé). Des communautés comme celle des Lost Boys and Girls ont des gardiens qui, sans avoir d'autorité officielle, exigent de savoir tout ce qui arrive à tous les membres du groupe. La présence de gardiens est à prévoir dans de nombreux projets de recherche en sciences sociales; en particulier ceux qui problématisent des sujets hautement politisés. De plus, comme Berger le rappelle: «Lorsque vous menez des recherches sur le terrain, toutes les complexités de l'accès sont décuplées si vous travaillez avec des populations engagées dans des activités illicites et sujettes à la stigmatisation», de même qu'avec celles considérées comme des «populations vulnérables» (Berger, 2003: 66, notre traduction).

Il nous a fallu beaucoup de temps pour gagner la confiance des gardiens dans le groupe. Parce qu'ils semblent avoir davantage de pou- 
voir social et d'ascendance dans la communauté, ils peuvent faciliter ou entraver l'accès à celle-ci et ainsi nuire considérablement au processus de recherche. Or, dans ce cas-ci, ils l'ont plutôt enrichi. Même si ces gardiens nous ont parfois limitée dans nos recherches, c'est en les côtoyant de près que de nouvelles questions, de nouveaux thèmes et de nouvelles informations ont émergé. Ils nous ont aidée à trouver notre place dans ce projet, en nous permettant de mieux comprendre les dynamiques hiérarchiques dans le groupe, sa culture et ses façons de s'organiser. En somme, leur participation à la planification du projet, aux groupes de discussion et aux entrevues était essentielle pour assurer l'accès aux autres membres du groupe parce qu'elle validait l'acceptabilité du projet.

Les entretiens avec de tels individus sont toujours délicats sur le plan éthique, puisque l'on évoque des expériences traumatisantes dans le passé des personnes interrogées. Dans le cas présent, cependant, il semble que les avantages potentiels du processus de recherche et les retombées involontaires que les entretiens ont eues sur les participants l'ont emporté sur les risques. Le processus leur permettant de discuter de leur passé, de leurs expériences, et de réfléchir sur la façon dont ils se sont débrouillés depuis leur migration au Canada a été décrit par eux comme une grande «délivrance». La majorité des répondants ont mentionné qu'ils parlaient de leurs expériences pour la première fois depuis leur arrivée au Canada. Sans doute, parler du passé s'est avéré ici cathartique, ou, comme plusieurs l'ont dit (surtout les femmes), une délivrance. À maintes reprises, les participants ont eu les larmes aux yeux en se rappelant la perte d'un être cher, les mauvais traitements subis ou la discrimination raciale ou sexuelle qu'ils ont réussi à surmonter. Parmi les femmes du groupe, plusieurs ont dit que le fait même d'être au Canada leur donnait un pouvoir et une voix qu'elles n'auraient jamais eus au Soudan:

Si j'étais au Soudan et qu'il y avait ce genre de possibilité d'un entretien, je ne pense pas que quiconque m'inviterait ici pour être interviewée. Ce serait tous des hommes, alors c'est différent au Soudan et ici... nous ne serions pas en train de parler en ce moment. (Notre traduction)

Dans les pages suivantes, nous exposons les résultats préliminaires de ce qui a donné un sens à leur expérience de réinstallation au Canada. Les résultats sont présentés suivant les thèmes qui ont émergé lors des rencontres de groupes et des entrevues individuelles. 


\section{Résultats préliminaires}

La «bonne voie» pour devenir un «bon » citoyen

Les individus interviewés ont fait part de plusieurs facteurs de protection qui les ont aidés depuis leur arrivée au Canada; un thème qui a permis de dégager d'extraordinaires similitudes au sein du groupe. Une majorité de répondants a rapporté que le respect des lois avait été le facteur de protection le plus utile pour devenir de «bons citoyens»:

Ce qui m'a protégé ici ce sont les règles déjà établies par le gouvernement canadien. Vous devez vous respecter et vous devez respecter les autres et c'est comme ça que tu dois faire, comme ne pas participer à la perpétration de crimes ou prendre un mauvais chemin. La loi établie par le gouvernement est la principale protection pour moi. (Kaleb [homme]; notre traduction)

Plusieurs Lost Boys and Girls disent se tenir loin de tentations comme la criminalité et l'argent facile, ce que les commentaires de Taye illustrent:

Le mauvais chemin, c'est que vous venez ici et il y a un moyen facile de gagner de l'argent... et si vous allez sur cette voie de l'argent facile, je ne crois pas que ce soit une bonne vie. Comme être un trafiquant de drogue, se saouler et se battre avec des gens. Ce n'est pas bien parce que vous considérez la liberté dans le mauvais sens. Vous vous saoulez et vous faites n'importe quoi parce que vous êtes libres, mais non, ce n'est pas la bonne façon de vivre. Vous êtes libres d'exprimer vos idées, vous êtes libres de vivre dans ce pays, mais vous n'êtes pas libres de provoquer de la violence. (Notre traduction)

Pour plusieurs répondants, les perspectives qui se présentent avec la drogue, l'alcool et la prostitution peuvent en emmener plus d'un à s'écarter de la «bonne voie». Cette mauvaise gestion de la liberté peut avoir des effets néfastes sur leur aptitude à se gouverner et à devenir des citoyens canadiens honnêtes, tout comme sur leur nouveau pouvoir d'agir. Mais ils ne tiennent pas leur pouvoir pour acquis, pas plus qu'ils ne le voient comme permanent. Ce dernier varie, et selon les individus, la liberté ou le pouvoir peuvent s'avérer inutiles, comme Kaleb le mentionne: «Certaines personnes pensent que si elles ont du pouvoir, elles peuvent tout maîtriser, mais un jour ce pouvoir peut s'effriter et elles seront à la merci des autres» (notre traduction). Pour certains, les nombreuses perspectives qui se présentent avec la liberté peuvent être trop difficiles à gérer, ce qui explique pourquoi plusieurs des partici- 
pants croient que certains de leurs pairs n'ont pas «réussi» aussi bien qu'eux:

Ici, il y a deux modes de vie: le chemin qui conduit à la mauvaise vie et le chemin qui mène à la bonne vie. Selon moi, il faut choisir la voie qui mènera à la bonne vie. Trouvez-vous un emploi et puis à partir de ce travail il y a de nombreux moyens d'acquérir des compétences. Vous développerez plus de compétences et vous gagnerez plus d'argent en faisant ce travail. C'est mieux que la mauvaise voie. (Taye; notre traduction)

Dans d'autres cas, c'est le fait de devoir recommencer à zéro qui représente un défi important pour les interviewés qui sentent qu'à cause de cela, ils ont dû travailler fort pour se reconstruire une identité dans cette nouvelle culture:

Je pense que la force qui me vient de là-bas (la guerre) m'a aidé à être patient avec ma vie qui repart à zéro. Mais ce n'est pas parce que je suis un homme... c'est grâce à ce que j'ai appris. Il y a des filles qui sont parties de rien, mais elles sont patientes car elles recommencent leur vie à zéro. Vous vous construisez à partir de rien, puis vous vous développez petit à petit, vous venez ici et vous vous développez sans les bases que vous aviez avant. (Taye; notre traduction)

\section{Débrouillardise et perspectives d'avenir}

Pour la plupart des personnes interrogées, apprendre à s'orienter dans le dédale des systèmes d'organisation sociale tant au Canada que dans les camps de réfugiés a joué un rôle crucial dans le remodelage de leur identité, de leur capacité d'action et a même influé sur comment ils ont réussi à venir au Canada. Les entretiens individuels montrent que les aptitudes des Lost Boys and Girls pour apprendre à s'orienter ont effectivement été cultivées dans les camps de réfugiés, bien avant leur arrivée au Canada. La question de leur âge illustre bien ceci. La majorité des répondants ne connaissent pas leur véritable date de naissance et ils ont rapidement appris qu'ils devaient répondre à certains critères de réinstallation pour venir s'établir au Canada. Pour cette raison, plusieurs nous ont raconté que leur «âge canadien» diffère de plusieurs années de leur âge véritable et que plusieurs d'entre eux ont déclaré être nés un $1^{\text {er }}$ janvier. Un participant, Mack, raconte que la première fois qu'il a rempli sa demande pour la réinstallation, elle lui a été refusée en raison de son trop jeune âge. Trois mois plus tard, il a présenté une 
nouvelle demande, affirmant avoir 21 ans. Comme il ne voulait pas oublier son anniversaire, il a choisi le $1^{\text {er }}$ janvier 1978 comme date de naissance - un anniversaire que partagent de nombreux répondants. Selon lui, ceux qui souhaitent venir au Canada et ignorent leur date de naissance ou sont trop jeunes vont échouer au processus d'entrevue: «J'ai donc manqué cette chance, mais pas la suivante... je me suis exercé... à être plus âgé. Je leur ai dit: "J'ai 21 ans" et ils ont dit: "Ok, vous êtes admissible." C'est là que j'ai eu ma chance!» (notre traduction).

Nous avons conclu que ces compétences pour apprendre à s'orienter ont été acquises dans les circonstances désespérées des camps de réfugiés, qui obligent ces jeunes à développer un sens de débrouillardise et des techniques de survie remarquables qu'ils emploient ensuite au Canada. Ils y ont recours de manière limitée, mais non négligeable, dans leur vie quotidienne en cultivant une attitude entrepreneuriale envers le travail et l'éducation, l'aide sociale, les prêts étudiants, les bourses d'études, l'Église, l'apprentissage des langues, ou les nombreux systèmes gouvernementaux auxquels ils ont recours.

Lavenir est avant tout le thème de discussion principal lorsqu' ils sont ensemble. En faisant cela, ils transcendent en quelque sorte les risques en misant sur les possibilités à exploiter, et donnent un sens à leur passé, transformant le risque en débouchés. Leur réticence à parler du passé s'explique surtout par ce qu'ils nomment «le privilège» de venir au Canada, la culpabilité du survivant et la responsabilité de réussir. Ils préfèrent concentrer leur énergie sur le présent, pour devenir des citoyens canadiens respectables et connaître une meilleure vie que celle que leur famille a connue.

\section{La culpabilité du survivant et la responsabilité envers le pays d'origine}

La majorité des interviewés sont animés d'un profond sentiment de responsabilité envers leurs compatriotes restés au Soudan ou les réfugiés des camps des pays voisins, et ce facteur peut favoriser la gestion des risques auxquels ils sont exposés. Ce sentiment de responsabilité les incite à «redonner» à ceux qui sont restés derrière, que ce soit financièrement, sur le plan de l'éducation ou d'une autre manière. Bien que la majorité des répondants ait déclaré ne pas avoir l'intention de retourner s'établir au Soudan, plusieurs ont l'intention de donner en retour d'une façon ou d'une autre. 
Les entrevues ont aussi levé le voile sur l'écrasant sentiment de culpabilité du survivant que semblent éprouver les répondants. Ceux-ci ont indiqué que ce sentiment les motive toutefois à faire de leur mieux au Canada afin de rendre leurs proches fiers, qu'ils soient ou non réunis avec eux. Par exemple, même si elle admet que tout dépend de chaque individu, Kate indique qu'il est dangereux pour une personne d'oublier les horreurs de la guerre et ce qu'elle a vécu, car elle risque aussi d'oublier que d'autres perspectives s'ouvrent à elle au Canada.

Lorsque les premiers chèques de paie d'Akilhah ont été directement déposés dans son compte de banque, elle était désorientée et bouleversée par ce salaire bimensuel de 1000 \$: «Quand j'ai vu mon argent, je l'ai envoyé à mes parents... Maintenant, je me sens mal parce que j'envoie tout mon argent, mais je ne ressens plus de culpabilité» (notre traduction).

Abigail envoie également de 200 à 300 \$ à sa famille mensuellement: «Beaucoup de Lost Boys le font, la plupart d'entre eux le font, et c'est important, ça l'est. C'est bien d'aider. Ce n'est pas comme si nous devions le faire, ce n'est pas comme ça, mais nous choisissons de le faire, pour les aider» (notre traduction). Quand nous l'interrogeons au sujet des filles qui envoient de l'argent, elle nous répond: «Un grand nombre de filles le font aussi. Elles envoient de l'argent à leurs frères, à leurs mères. Un grand nombre de Soudanais qui ont des proches au pays, ils envoient de l'argent pour eux. S'ils ont besoin d'aide, je le ferai toujours» (notre traduction).

Certaines personnes interrogées sont très sceptiques ou méfiantes quand il est question d'envoyer de l'argent pour aider les autres au pays, parce qu'elles craignent la corruption ou ignorent si leur argent se rend bien à destination, comme l'affirme Abok:

Je dois le voir pour le croire, qu'ils le font pour eux. Parce que parfois, quelqu'un va dire une chose et puis ils vont faire une autre. La seule charité qui m'intéresserait vraiment, ou à laquelle je pourrais croire, c'est celle qui prendrait le parti d'aider ceux qui n'ont pas de parents. Parce que je l'ai vraiment vu, comment la vie peut être difficile sans les parents pour nous soutenir. (Notre traduction)

Malgré cette attitude envers l'envoi d'argent au Soudan, la plupart des Lost Boys and Girls sentent une grande responsabilité d'agir pour le bien et de donner en retour, et en assumant cela, ils atténuent leur sentiment de culpabilité d'être sortis des camps de réfugiés et de s'être forgé une vie meilleure au Canada. 


\section{Les familles de substitution}

De nombreux répondants ont été «adoptés» par une famille canadienne. Selon eux, cette famille a contribué à leur propre bien-être (ou capital affectif) et à leur réussite au Canada, et s'est avérée un autre facteur de protection en leur faveur. Plusieurs Lost Boys vivent dans l'incertitude permanente de savoir où se trouvent les membres de leur famille et si ceux-ci sont vivants (Luster et al., 2008). Par exemple, Mack a été séparé de sa famille à l'âge de neuf ans et n'a pu être réuni avec sa mère biologique pour la première fois en 20 ans que quelques jours avant notre rencontre. Cependant, par l'entremise de son Église, il avait rencontré une autre «maman», avec qui il demeure maintenant et qui l'aide à élever son enfant. Comme Mack l'explique, ils sont maintenant une famille. Calah aussi a rencontré, par son Église, une seconde mère qui a payé son loyer alors qu'elle était démunie, qui lui promet de l'aider à payer ses frais d'inscription au collège si elle obtient des résultats satisfaisants à l'école secondaire:

Elle est presque comme une mère... parce que je n'ai pas de maman ici et elle maide toujours quand je suis dans une situation difficile ou lorsque quelque chose me peine ou me tourmente. C'est vraiment drôle parce que parfois je l'appelle «maman» et elle rit tout simplement... Ces dernières années, je lui ai donné une carte pour la fête des Mères. Quand je la lui ai donnée, elle s'est mise à pleurer très fort. (Notre traduction)

Pour les répondants qui ont été adoptés non officiellement par un individu, une famille, ou même par des membres de leur Église, cette adoption les a aidés à réussir leur réinstallation au Canada. C'est à ces familles qu'ils attribuent leur succès, leur motivation et leur confiance pour réussir à l'école, au travail et pour prendre de bonnes décisions dans la vie.

\section{Le capital religieux et social}

Les récits des participants offrent divers exemples de leur capital religieux et social et même si les répondants, de leurs propres dires, n'abordent généralement pas leur passé (sauf dans le cadre de cette recherche), celui-ci semble néanmoins être la source d'importantes leçons de vie, d'où ils puisent une assurance et une force intérieure:

Je crois que prendre soin de mon garçon, et je pense que d'avoir été seul en tant qu'enfant soldat, a fait de moi la personne que je suis maintenant, 
parce que si ce n'était pas ça... en tant qu'enfant soldat, laissé à moi-même, je pense que ça m’a préparé à prendre soin de mon enfant. J'ai vécu tout et n'importe quoi pour prendre soin de cet enfant. (Mack; notre traduction)

Tous les individus interrogés dans le cadre de cette recherche croient fermement qu'ils ont survécu grâce à l'appui qu'ils ont reçu de leurs amis, de Dieu, et de leur communauté religieuse. Aucun d'entre eux n'attribue leur succès uniquement à la chance ou à leur détermination individuelle. Cela peut être dû au fait qu'une grande partie du soutien social et des organismes de parrainage qui accueillent les réfugiés à leur arrivée est basée sur la foi (principalement chrétienne), et que dès l'aéroport, ce sont des groupes religieux qui prennent en charge leur intégration dans la société d'accueil (Shandy, 2004: 22). La plupart des répondants attribuent donc une grande part de leur succès au Canada au soutien reçu de leur Église. Cette tendance est appuyée par des études antérieures qui dégagent, entre autres, une bonne santé spirituelle, une bonne estime de soi, de fortes croyances religieuses, de bonnes relations avec les pairs, une identité culturelle positive, de même qu'un dense réseau de soutien social (capital social et humain). Tous ces facteurs sont des éléments qui renforcent les valeurs culturelles, familiales et religieuses (Hunt et al., 2002).

Des études antérieures sur comment les Lost Boys perçoivent l'avenir, les possibilités à exploiter et le soi collectif (Goodman, 2004) appuient les conclusions tirées de leurs témoignages dans cette étude. Les répondants soulignent également l'importance du soutien social (c'est-à-dire le capital social) dans leur réussite au Canada. Cette constatation confirme l'importance des réseaux sociaux, signalée par $M$. Bordet (2001), qui remarque aussi une tendance à l'ethnicisation des liens sociaux lorsque des groupes de jeunes immigrants se rassemblent dans les grandes villes, en particulier dans les quartiers de logements sociaux. Bordet assimile ce type de réseautage social à une sorte de microsociété où les immigrants développent un sentiment d'appartenance à une communauté. C'est le cas chez les Lost Boys and Girls de Calgary et d'Edmonton. La recherche sur le terrain a permis de découvrir plusieurs illustrations de cette microsociété, dont celle des Lost Boys and Girls qui souvent se viennent en aide mutuellement pour assurer leur éducation: un garçon travaille pour payer les frais d'inscription d'un semestre ou deux à son ami, puis l'autre garçon quitte l'école pendant quelques semestres, pour rendre la pareille au premier étudiant. Ou encore, si un individu est en difficultés financières, la communauté met ses fonds en 
commun pour lui permettre de payer son loyer. Plus tard, il retournera la faveur d'une manière ou d'une autre. Quelques hommes ont voyagé au Soudan pour renouer avec leur famille, construire des écoles et, dans un cas, filmer un documentaire. Pour chacun d'eux, le coûteux voyage a été rendu possible grâce à la participation de la communauté. Un tel système pourrait être comparé à un système pyramidal, mais dont tous profiteraient. En somme, il semble qu'un ensemble de facteurs de protection, y compris des mécanismes collectifs de survie, un capital religieux et émotionnel, ainsi que de solides réseaux sociaux, jouent en faveur des Lost Boys and Girls.

\section{Étiquetage}

Lorsque ces jeunes étaient dans les camps de réfugiés, ils ont été étiquetés "The Lost Boys», une étiquette qui, grâce à la reconnaissance internationale et aux enquêtes journalistiques qui ont fait connaître leur histoire, englobe désormais l'ensemble plus large des The Lost Boys and Girls du Soudan. Cependant, ce terme a acquis encore plus de notoriété parce que les Lost Boys and Girls se sont eux-mêmes identifiés comme tels. Bien que l'étiquetage soit souvent vu péjorativement dans le domaine de la criminologie, puisqu'il peut avoir d'énormes conséquences et compromettre la guérison des victimes ou des auteurs d'actes criminels, dans le cas présent, le fait même d'étiqueter ce groupe de réfugiés s'est avéré bénéfique pour eux. Les Lost Boys and Girls estiment que cette étiquette leur a valu une plus grande visibilité au sein des communautés locales et internationales, validant davantage la microsociété décrite par Bordet, où un groupe parvient, dans ce cas-ci grâce à l'étiquetage, à se construire une solide identité communautaire.

Quant à la reconnaissance en tant que communauté, les répondants ont généralement l'impression que l'étiquette contribue à l'organisation et l'identification du groupe et permet de construire un capital social étendu. Mack confirme cette idée en expliquant que les gens considèrent les Lost Boys and Girls comme une communauté ayant un objectif commun: acquérir des compétences et procurer de l'aide au peuple du Soudan pour empêcher que d'autres enfants connaissent des situations similaires à celles qu'ils ont vécues. Il explique aussi qu'avec cette étiquette, ils sont vus comme un groupe auquel on peut faire appel au besoin, et un groupe qui a lui-même reçu le soutien d'organisations, à savoir leurs Églises. 
Malgré les avantages que procure cette étiquette, celle-ci provoque également un certain ressentiment chez quelques-uns dans le groupe et peut parfois s'avérer un facteur de division plutôt que de cohésion. Cette division s'est manifestée entre les sexes et les niveaux hiérarchiques. Si elle a, aux dires de plusieurs d'entre eux, conscientisé le public à leur égard et permis au groupe de gagner en reconnaissance, elle ne les a pas nécessairement aidés individuellement. En fait, certains des participants estiment que l'étiquette a seulement profité à quelques-uns d'entre eux, soit les dirigeants du groupe (parmi lesquels il n'y a aucune femme). Il semble effectivement que l'étiquette puisse être fondée sur le sexe, comme Calah l'illustre:

C'est tout simplement fou parfois. Prends ces gars qui ont fait un documentaire. Je ne l'ai pas encore vu. Ils sont toujours occupés... Ils sont toujours occupés à faire une foule de choses, et à essayer d'attirer l'attention [...] et ils ne parlent généralement pas de nous, les filles. C'est vraiment frustrant, je pense. (Notre traduction)

\section{Conclusion}

Cet article a exploré comment plusieurs Lost Boys and Girls of Sudan considèrent avoir réussi leur réinstallation au Canada par l'entremise d'objectifs tels que devenir d'honnêtes citoyens, conserver une responsabilité envers leur pays, créer des liens avec une famille de substitution et d'autres réseaux de soutien, et tirer avantage de l'étiquette de «Lost Boys and Girls».

D'une part, nos résultats préliminaires suggèrent que les enfants séparés qui cherchent refuge au Canada rencontrent des obstacles psychosociaux particuliers et acquièrent des compétences uniques en raison de leur expérience de la guerre. Les problèmes auxquels ils se heurtent sont en effet très différents de ceux vécus en zone de paix par les jeunes Canadiens moyens. D'autre part, les résultats dressent aussi un tableau complexe de ce qui contribue, selon le propre témoignage des répondants, à la réussite de leur réinstallation. Pour se réinventer, ils canalisent leur capital social, religieux et affectif ainsi que leur expérience des ravages de la guerre pour les transformer en facteurs de motivation parce qu'ils se sentent responsables envers leurs nouvelles familles de substitution, la communauté des Lost Boys and Girls et ceux qui sont toujours au pays, ce qui les pousse à donner le meilleur d'euxmêmes. 
Ce bref survol d'une vaste étude montre clairement les obstacles particuliers qu'affrontent les mineurs séparés. Plus important encore, il montre qu'en explorant les nombreuses possibilités qu'ils réussissent à exploiter, nous pouvons élaborer de nouvelles stratégies pour mieux protéger ces jeunes et minimiser les risques auxquels ils sont exposés dès leur arrivée dans un pays étranger. Pour ce faire, il est essentiel de procéder à un partage des responsabilités et de mieux maitriser l'afflux des mineurs séparés qui viennent au Canada. Mais il est aussi important de reconnaître que sans une évaluation convenable des besoins particuliers des jeunes réfugiés provenant de zones de violence politique et de conflits armés, la prévention du crime et les réponses sociales concernant ces enfants risquent de ne pas être efficaces. Les Lost Boys and Girls of Sudan ne sont qu'un groupe parmi une large population de jeunes réfugiés, mais en fait, ce sont eux les experts qui peuvent nous en apprendre le plus et nous seconder dans nos efforts pour élaborer des stratégies cohérentes, innovatrices et saines visant l'intégration réussie des jeunes dans la toile colorée et diversifiée qu'est le Canada.

\section{Références}

Ali, M., Taraban, S., \& Gill, J. (2002). Unaccompanied / Separated Children Seeking Refugee Status in Ontario : A Review of Documented Policies and Practice. Toronto: Centre of Excellence for Research on Immigration and Settlement (CERIS).

Anisef, P., \& Kilbride, K. M. (2001). To Build on Hope: Overcoming the Challenges Facing Newcomer Youth at Risk in Ontario. Toronto: CERIS.

Anisef, P., Kilbride, K. M., Baichman-Anisef, E., \& Khattar, R. (2001). Between Two Worlds: The Experiences and Concerns of Immigrant Youth in Ontario. Toronto : CERIS.

Beiser, M., Dian, R., Gotowiec, A., Hyman, I., \& Vu, N. (1995). Immigrant and Refugee Children in Canada. Canadian Journal of Psychiatry, 40, 67-72.

Berger, M. T. (2003). Dealing with Difficult Gatekeepers, Vulnerable Populations and "Hooks" that Go Awry: An Access Story. In M. T. Berger, J. Bell \& M. Feldman (Eds.), Gaining Access: A Practical and Theoretical Guide for Qualitative Researchers (65-68). New York: Altamira Press.

Bordet, J. (2001). Les microsociétés des jeunes dans les quartiers d'habitat social. Revue européenne des migrations internationales, 17 (2), 169-174.

Burisk, R. (1989). Political Decision Making and Ecological Models of Delinquency: Conflict and Consensus. In S. Messner, M. Krohn \& A. Liska (Eds.), Theoretical Integration in the Study of Deviance and Crime: Problems and Prospects (105-117). Albany: SUNY Press.

Debora, N., Liwiski, N., Palacios, C., \& Gardinier, M. (1996). Traumatized Children: Helping Child Victims of Violence. In Y. Danieli, N. Rodeley \& 
L. Weisaith (Eds.), International Responses to Traumatic Stress (327-346). Amityvlle, NY: Baywood Publishing Company.

Duncan, J. (2001). Sudanese "Lost Boys" in the United States: Adjustment after Six Month. United States Catholic Conference, Washington, DC. Disponible sur www.brycs.org/documents/upload/lostboys.pdf

Elgersman, S. (2007). Unaccompanied and Separated Minors as Refugee Claimants. Parliamentary Information and Research Service Library of Parliament, Ottawa, Ont.

Frostfeldt, A. (2002). Lost Sudanese Boys Prioritized: Girls Left Behind in Kakuma Refugee Camp. Mars 2000, 7 (1). Disponible sur www.essex.ac.uk/armedcon. html

Geltman, P., Grant-Knight, W., Mehta, S., Lloyd-Travaglini, C., Lustig, S., Landgraf, J., \& Wise. P. (2005). The Lost Boys of Sudan: Functional and Behavioral Health of Unaccompanied Refugee Minors Resettled in the United States. Archives Pediatric Adolescence Medicine, 159, 585-591.

Gladwell, M. (2008). Outliers: The Story of Success. New York, Little, Brown \& Company.

Goodman, J. (2004). Coping with Trauma and Hardship Among Unaccompanied Refugee Youths from Sudan. Qualitative Health Research, 14 (9), 11771196.

Hagen, J. (1994). Crime and Disrepute. Thousand Oaks: Pine Forge Press.

Hunt, D., Morland, L., Barocas, R., Huckans, M., \& Caal, S. (2002). Understanding, Preventing and Treating Problem Behaviours among Immigrant and Refugee Youth. Virginia: Centre for Multicultural Human Services.

Luster, T., Qin, D., Bates, L., Johnson, D., \& Rana, M. (2008). The Lost Boys of Sudan: Ambiguous Loss, Search for Family, and Re-establishing relationships with Family Members. Family Relations, 57 (octobre), 444-456.

Marfleet, P. (2006). Refugees in a Global Era. New York: Palgrave.

Matheson, I. (7 juin 2002). The Lost Girls of Sudan. BBC News Africa.

McKelvey, T. (3 octobre 2003). Where are the Girls? Disponible sur www.slate. com.

Moser, A. (2000). Young People in Migration: A Challenge for Social Services. Young People in Migration: A Challenge for Social Services. (Report Proceedings on the meeting of the International Social Service Council,"Open Day” May $25^{\text {th }}$ 2000). Genève: International Social Service.

Newland, K. (2004). Migration and Mobility and how this Movement affects Women. Seeking Protection: Women in Asylum or Refugee Resettlement Processes (Report CM/MMW/2003/EP8). United Nations Division for the Advancement of Women, 2-13.

Nhial, A., \& Mills, D. (2004). Lost Boy No More: A True Story of Survival and Salvation. Nashville: Broadman \& Holman.

Refugees International (2002). Do Not Forget the Lost Girls of Sudan. Refugees International, A Powerful Voice for Lifesaving Action (ReliefWeb report). Disponible sur http://reliefweb.int/sites/reliefweb.int/files/reliefweb_pdf/ node-95539.pdf 
Schweitzer, R., Melville, F., Steel, Z., \& Lacherez, P. (2006). Trauma, Postmigration Living Difficulties, and Social support as Predictors of Psychological Adjustment in Resettled Sudanese Refugees. Australian and New Zealand Journal of Psychiatry, 40, 179-187.

Shandy, D. (2004). Christianity and Southern Sudanese in the United States. Department of Anthropology, McCalaster College, in Lost Boys Film Educational Study Guide, 21-24. Disponible sur www.lostboysfilm.com/assets/ LostBoys-EducationGuide.pdf

Shaw, C. \& McKay, H. (1969). Juvenile Delinquency and Urban Areas. Chicago: University of Chicago Press.

Stowell, J. O., \& Martinez, R. Jr. (2007). Displaced, dispossessed, or lawless? Examining the link between ethnicity, immigration, and violence. Aggression and Violent Behaviour, 12, 564-581.

UNHCR (2002). Why the Difference? Refugees Magazine, 126.

US Agency for International Development (USAID) (2004). Youth and Conflict. Washington, DC: Office of Conflict Management and Mitigation.

World Health Organization (Krug E., Dahlberg L., Mercy J., Zwi, A., \& Lozano, R. (2002). World Report on Violence and Health. Geneva: World Health Organization. Disponible sur www.who.int/violence_injury_prevention/ violence/world_report/en/

Wouk, J., Yu, S., Roach, L., Thomson, J., \& Harris. A. (2006). Unaccompanied/ separated minors and Refugee Protection in Canada: Filling information Gaps. Refuge, 23 (6), 125-138.

Yau, M. (1995). Refugee Students in Toronto Schools: An Exploratory Study. Toronto: Research Department: Toronto Board of Education.

ABSTRACT - Studies of young survivors of armed conflict and political violence represent a growing area of interest within the field of criminology. In this regard, this article highlights how a handful of protective factors have favoured the resettlement in Canada of about thirty "Lost Boys and Girls" of Sudan, refugee children separated from their families because of the war in South Sudan. Preliminary analysis of individual interviews and focus groups conducted with these youth help to shed light on the role of protective factors that they themselves have identified, including their desire to become "good citizens", their resourcefulness and future prospects, survivor guilt and sense of responsibility to their country of origin, surrogate families, their social, religious and emotional capital, and even the label of "Lost Boys and Girls".

KEYWORDS - Lost Boys and Girls of Sudan, separated/unaccompanied refugees, criminology, protective factors, immigration, Canada.

RESUMEN - Los estudios sobre jóvenes sobrevivientes de conflictos armados y de violencia política constituyen una rama de interés creciente en criminología. El presente artículo analiza, en ese sentido, un conjunto de factores que se estima facilitó la reinstalación en Canadá de una treintena de "niños y niñas perdidos" de Sudán, es decir, de jóvenes refugiados separados de sus familias a causa de la guerra en el sur 
de Sudán. El análisis preliminar de entrevistas individuales y discusiones de grupo realizadas con esos jóvenes destaca el papel de algunos factores de protección identificados por ellos mismos, por ejemplo el deseo de convertirse en "buenos ciudadanos", su espíritu emprendedor y sus perspectivas a futuro, la culpa por haber sobrevivido y el sentimiento de responsabilidad respecto de su país de origen, sus familias sustitutas, su capital social, afectivo y religioso, e incluso la etiqueta de "niños y niñas perdidos".

PALABRAS CLAVE - Niños y niñas perdidos de Sudán, menores separados o no acompañados, protección, criminología, inmigración, Canadá. 